\title{
Integrative genomics identifies new genes associated with severe COPD and emphysema
}

Phuwanat Sakornsakolpat ${ }^{1,2}$, Jarrett D. Morrow ${ }^{1}$, Peter J. Castaldi ${ }^{1,3}$, Craig P. Hersh ${ }^{1,4}$, Yohan Bossé ${ }^{5}$, Edwin K. Silverman ${ }^{1,4}$, Ani Manichaikul ${ }^{6}$ and Michael H. Cho ${ }^{1,4^{*}}$

\begin{abstract}
Background: Genome-wide association studies have identified several genetic risk loci for severe chronic obstructive pulmonary disease (COPD) and emphysema. However, these studies do not fully explain disease heritability and in most cases, fail to implicate specific genes. Integrative methods that combine gene expression data with GWAS can provide more power in discovering disease-associated genes and give mechanistic insight into regulated genes.
\end{abstract}

Methods: We applied a recently described method that imputes gene expression using reference transcriptome data to genome-wide association studies for two phenotypes (severe COPD and quantitative emphysema) and blood and lung tissue gene expression datasets. We further tested the potential causality of individual genes using multi-variant colocalization.

Results: We identified seven genes significantly associated with severe COPD, and five genes significantly associated with quantitative emphysema in whole blood or lung. We validated results in independent transcriptome databases and confirmed colocalization signals for PSMA4, EGLN2, WNT3, DCBLD1, and LILRA3. Three of these genes were not located within previously reported GWAS loci for either phenotype. We also identified genetically driven pathways, including those related to immune regulation.

Conclusions: An integrative analysis of GWAS and gene expression identified novel associations with severe COPD and quantitative emphysema, and also suggested disease-associated genes in known COPD susceptibility loci.

Trial registration: NCT00608764, Registry: ClinicalTrials.gov, Date of Enrollment of First Participant: November 2007, Date Registered: January 28, 2008 (retrospectively registered); NCT00292552, Registry: ClinicalTrials.gov, Date of Enrollment of First Participant: December 2005, Date Registered: February 14, 2006 (retrospectively registered).

Keywords: Chronic obstructive pulmonary disease, Emphysema, Genome-wide association studies, Gene expression

\footnotetext{
*Correspondence: remhc@channing.harvard.edu

'Channing Division of Network Medicine, Department of Medicine, Brigham and Women's Hospital, 181 Longwood Avenue, Room 451, Boston, MA 02115, USA

${ }^{4}$ Division of Pulmonary and Critical Care Medicine, Department of Medicine, Brigham and Women's Hospital, 181 Longwood Avenue, Room 451, Boston, MA 02115, USA

Full list of author information is available at the end of the article
} 


\section{Background}

Chronic obstructive pulmonary disease (COPD) is characterized by irreversible airflow obstruction and is strongly influenced by genetic factors $[1,2]$. Genomewide association studies of COPD and related traits (e.g., emphysema) have revealed multiple genetic loci associated with disease risk [3-5]. Most loci identified by genome-wide association studies (GWAS) are regulatory, and do not directly alter the amino acid sequence.

Gene expression is arguably the most impactful and well-studied effect of regulatory genetic variation. GWAS loci are enriched for expression quantitative trait loci (eQTL), rendering it a potential link between genetic variant and biology of disease [6, 7]. The efforts of large cohort studies and consortia such as the GenotypeTissue Expression Project have discovered thousands of genetic variants associated with gene expression in multiple tissues. While most GWAS studies do not concomitantly measure gene expression, the strong relationship of genetic variation to gene expression allows one to use gene expression reference datasets to predict gene expression given a set of genotypes, and subsequently identify gene expression differences for a given phenotype. This approach has been implemented in software called S-PrediXcan and TWAS [8-10]. Aggregating information from variant level to infer genelevel associations increases the power to discover more genes at loci not previously implicated by GWAS and gives mechanistic insight regarding genes being regulated via disease-associated genetic variants $[7,11]$.

Despite the convention of naming a discovered locus for the nearest gene (e.g., HHIP), further study is needed to identify the specific gene(s) and variant(s) responsible for disease risk $[9,11,12]$. In identified COPD susceptibility loci, most loci contain multiple genes, and variants in these genes are correlated (in linkage disequilibrium). More than one gene in a locus may also play a role in disease pathogenesis, as seen in other complex diseases $[13,14]$. With recently developed methods and a growing amount of gene expression data made publicly available, integrating GWAS with known functional annotations of each variant (e.g., associated with gene expression) could highlight novel and biologically relevant genes for further evaluation.

We hypothesized that application of these integrative methods to specific phenotypes of COPD (severe disease and quantitative emphysema) would facilitate discovery of new gene-disease associations and elucidate the mechanism of gene in existing susceptibility loci. Specifically, we sought to identify genes and pathways genetically up- or down-regulated by phenotype-associated variants in tissue-specific reference datasets using S-PrediXcan and TWAS $[3,5]$, and to assess the potential causality of individual genes using multi-variant colocalization. ${ }^{1}$

\section{Methods}

Genome-wide association studies and meta-analysis

We used genome-wide association summary statistics for two phenotypes based on the same four cohorts. Demographic characteristics of individuals included in analyses of these two phenotypes are summarized in Tables 1 and 2. The four cohorts included individuals enrolled in Genetic Epidemiology of COPD (COPDGene, NCT00608764), Evaluation of COPD Longitudinally to Identify Predictive Surrogate Endpoints (ECLIPSE, SCO104960, NCT00292552), National Emphysema Treatment Trial (NETT) and Normative Aging Study (NAS), and GenKOLS (Genetics of COPD, Norway). Meta-analyses of these two phenotypes were published previously $[3,5]$. Severe COPD was defined by postspirometric measures of forced expiratory volume in 1st second $\left(\mathrm{FEV}_{1}\right)$ lower than $50 \%$ of predicted value and the ratio of $\mathrm{FEV}_{1}$ to forced vital capacity $\left(\mathrm{FEV}_{1} / \mathrm{FVC}\right)$ less than 0.7, excluding individuals with known severe alpha-1 antitrypsin deficiency. For quantitative emphysema, we produced the histogram of segmented CT chest images and used the percentage low attenuation area at - 950 Hounsfield units (HU) threshold (\%LAA950), and the $\mathrm{HU}$ at the 15 th percentile of the density histogram (Perc15) for the quantification of emphysema. A summary of our approach is shown in Fig. 1.

\section{Integration of GWAS and gene expression}

To integrate our GWAS and gene expression results, we used S-PrediXcan [10]. We included two relevant reference transcriptome databases in our analysis, whole blood from Depression Genes and Networks (DGNBlood) and lung tissue from Genotype-Tissue Expression consortium (GTEx-Lung). Details on prediction models and datasets used were provided in Additional file 1: Supplementary Methods. The ability of genetic variants to predict the expression of individual genes varies; only genes with significant prediction models were included in the analysis $(11,529$ genes for DGN-Blood and 6425 genes for GTEx-Lung). We accounted for multiple hypothesis testing using Bonferroni correction to determine statistical significance of gene-disease associations, resulting in $p$-value of $4.34 \times 10^{-6}$ and $7.78 \times 10^{-6}$ for DGN-Blood and GTEx-Lung, respectively.

\section{Validation in other reference transcriptome databases}

To determine whether our imputed gene expression was consistent in other datasets, we tested significant genes from DGN-Blood and GTEx-Lung in two independent reference transcriptome databases, GTEx for whole blood (GTEx-Blood) and the Lung-eQTL Consortium for lung tissue using S-PrediXcan and TWAS/FUSION (Additional file 1: Supplementary Methods). We considered an expression result to be validated if the direction 


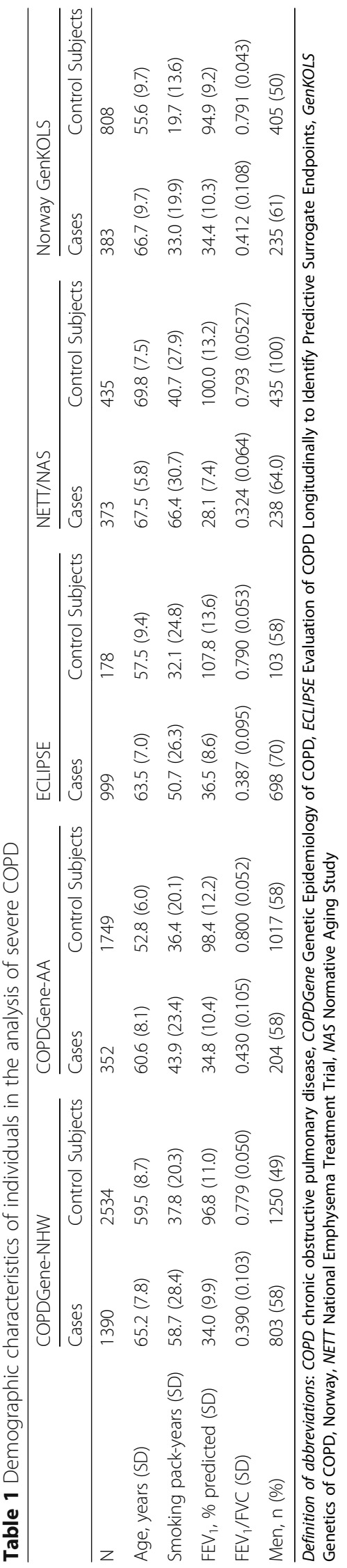




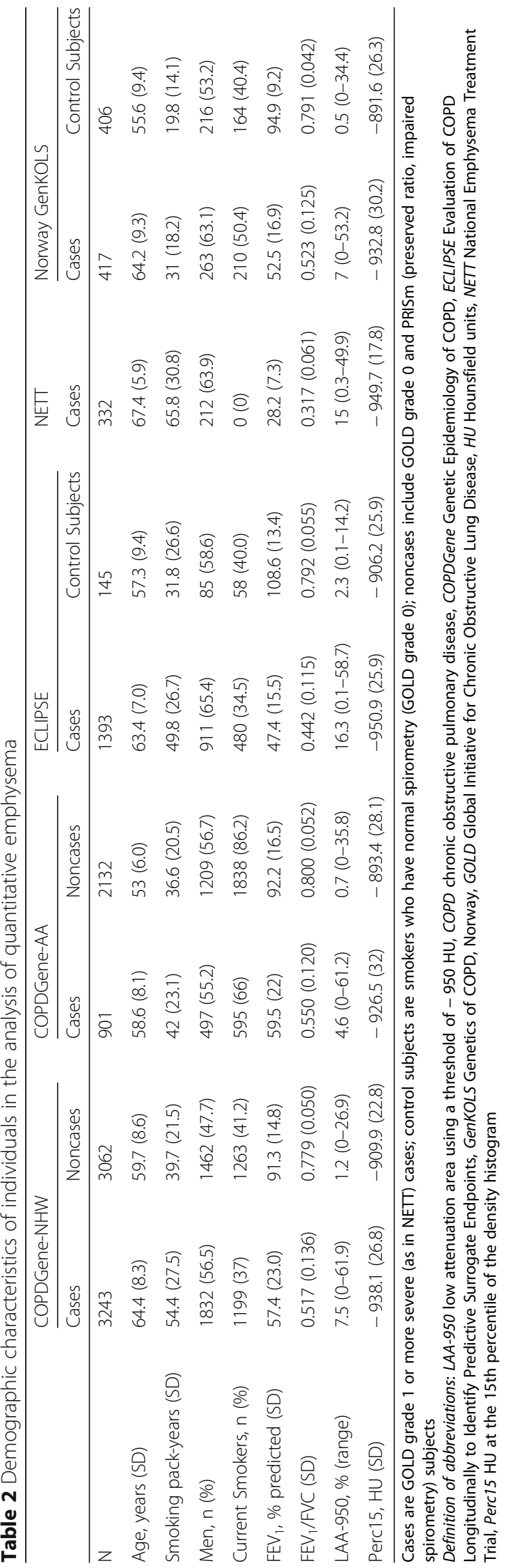




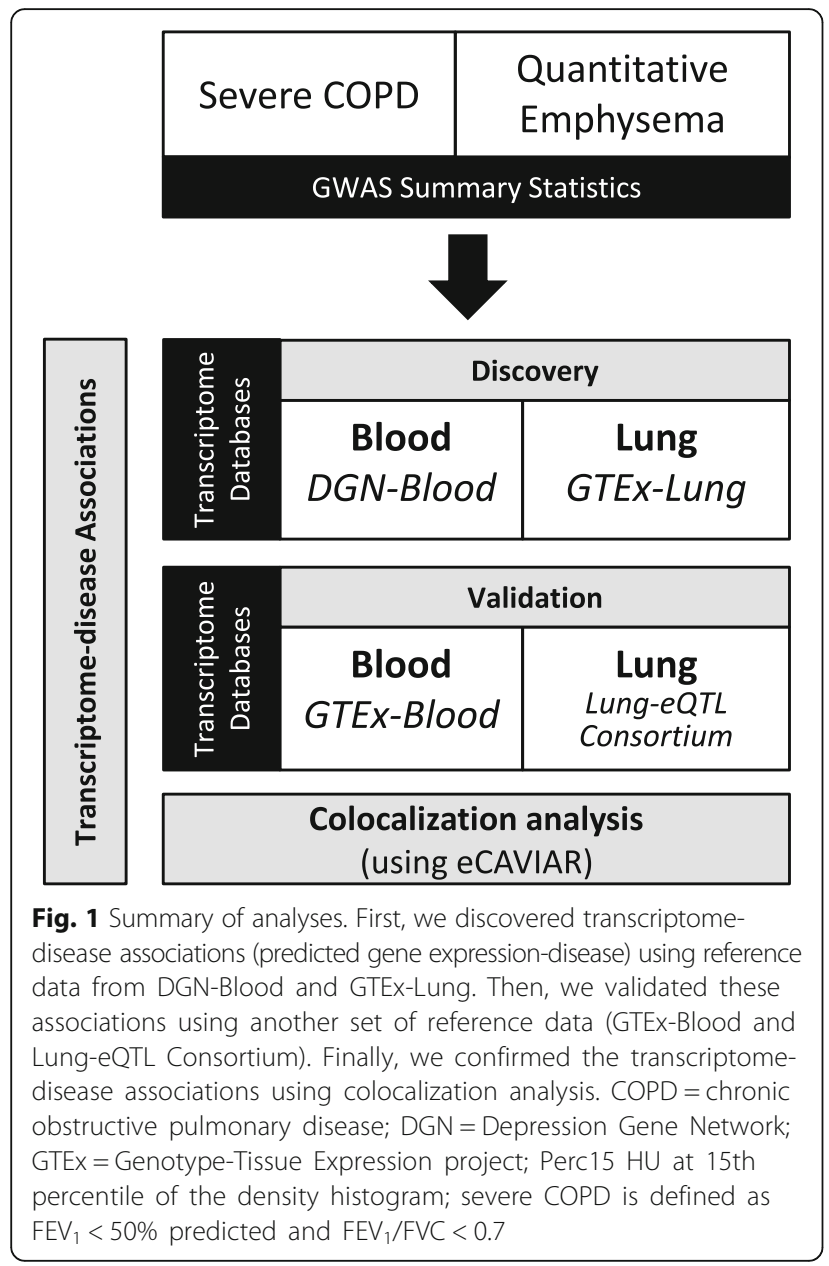

of effect was consistent and the Bonferroni-corrected $P$ value $<0.05$.

\section{Colocalization analysis using eCAVIAR}

Colocalization analysis estimates a posterior probability that a given variant or set of variants is causal for both the phenotype of interest (e.g., COPD) and expression level of a given gene. We used eCAVIAR (eQTL and GWAS Causal Variant Identification in Associated Regions), as it allows for multiple causal variants [15]. Details on parameters and procedures used in the analysis were present in Additional file 1: Supplementary Methods. Genes identified in whole blood were tested for colocalization using eQTL from GTEx-Blood while using GTEx-Lung and Lung-eQTL Consortium for lung tissue. The probability of a variant to be causal for a given gene in both datasets was determined by the colocalization posterior probability (CLPP) that approximates the posterior probability of a variant to be causal in GWAS and posterior probability of a variant to be causal in eQTL [15]. We also obtained functional annotations of colocalized variants in lung relevant cell types (Additional file 1: Supplementary Methods).

\section{Results}

\section{Severe COPD}

We first examined the association between severe COPD and imputed gene expression. Significant associations based on gene-based Bonferroni corrections for DGNBlood and GTEx-Lung are shown in Table 3 and Fig. 2.

In the whole blood reference dataset from DGN, we identified five significant genes: FAM13A in $4 \mathrm{q} 22$

Table 3 Result of association analysis between imputed gene expression and severe COPD and emphysema (\%LAA-950 and Perc15) with validation

\begin{tabular}{|c|c|c|c|c|c|c|c|}
\hline $\begin{array}{l}\text { Genetic } \\
\text { Loci }\end{array}$ & Phenotype & Gene & Tissue & $\begin{array}{l}\text { Discovery } \\
\text { Z score }\end{array}$ & $\begin{array}{l}\text { Discovery } \\
P \text { value }\end{array}$ & $\begin{array}{l}\text { Validation } \\
\text { Z score }\end{array}$ & $\begin{array}{l}\text { Validation } \\
P \text { value }\end{array}$ \\
\hline $4 q 22$ & Severe COPD & FAM13A & Blood & -5.46 & 4.80E-08 & NA & NA \\
\hline $4 q 22$ & Severe COPD & GPRIN3 & Lung & 4.95 & 7.40E-07 & 0.94 & $3.40 \mathrm{E}-01$ \\
\hline $5 q 11$ & Perc15 & ITGAI & Lung & -4.89 & 1.00E-06 & NA & NA \\
\hline $6 p 21$ & \%LAA-950 & ATF6B & Lung & -4.48 & 7.30E-06 & -1.33 & $1.80 \mathrm{E}-01$ \\
\hline $6 q 22$ & \%LAA-950 & DCBLD1 & Lung & 4.53 & 5.80E-06 & 3.81 & $1.40 \mathrm{E}-04$ \\
\hline $15 q 25$ & Severe COPD & HYKK & Blood & -8.33 & $8.20 \mathrm{E}-17$ & NA & NA \\
\hline $15 q 25$ & Severe COPD & PSMA4 & Blood & 7.62 & $2.50 \mathrm{E}-14$ & 7.57 & $3.80 \mathrm{E}-14$ \\
\hline $15 q 25$ & \%LAA-950 & HYKK & Blood & -5.64 & 1.70E-08 & NA & NA \\
\hline $15 q 25$ & \%LAA-950 & PSMA4 & Blood & 4.92 & 8.50E-07 & 5.1 & $3.40 \mathrm{E}-07$ \\
\hline $17 q 21$ & Severe COPD & WNT3 & Lung & 4.85 & $1.20 \mathrm{E}-06$ & 4.6 & 4.30E-06 \\
\hline $19 q 13$ & Severe COPD & EGLN2 & Blood & 4.89 & $1.00 \mathrm{E}-06$ & 4.35 & 1.30E-05 \\
\hline $19 q 13$ & Severe COPD & $R A B 4 B$ & Blood & -4.78 & $1.70 \mathrm{E}-06$ & -3.82 & 1.30E-04 \\
\hline $19 q 13^{a}$ & \%LAA-950 & LILRA3 & Blood & -4.62 & 3.80E-06 & -4.13 & 3.60E-05 \\
\hline
\end{tabular}

Lung = Genotype Tissue Expression (GTEx) consortium lung, Blood = Depression Genes and Networks (DGN) blood, NA = prediction model not available in the validation dataset (see Discussion)

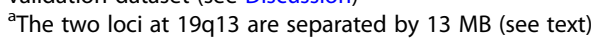



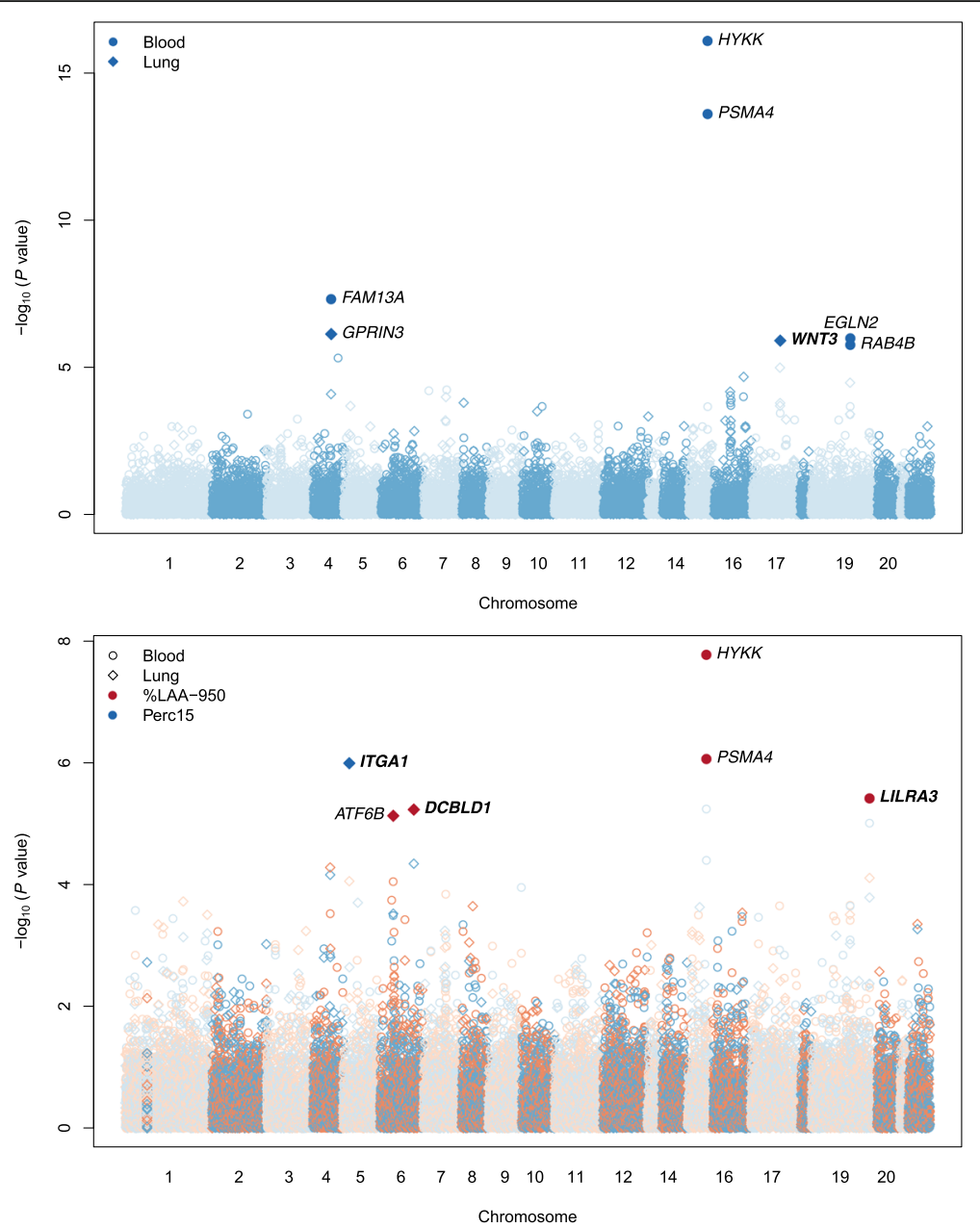

Fig. 2 Manhattan plots of associations of imputed gene expression and phenotypes (severe COPD in the upper panel; \%LAA-950 and Perc15 in the lower panel). Color indicates phenotypes and shape indicates tissue (see figure legend)

$\left(P=4.81 \times 10^{-8}\right), H Y K K$ and PSMA4 in $15 \mathrm{q} 25(P=$ $8.16 \times 10^{-17}$ and $2.47 \times 10^{-14}$, respectively), and EGLN2 and RAB4B in the $19 \mathrm{q} 13$ locus $\left(P=1.03 \times 10^{-6}\right.$ and $1.72 \times 10^{-6}$, respectively). All of these genes are located in COPD susceptibility loci previously reported in the literature $[4,16]$. In lung tissue, we identified two genome-wide significant genes, GPRIN3 in the 4q22 locus $\left(P=7.43 \times 10^{-7}\right)$ and WNT3 in the $17 \mathrm{q} 21$ locus $\left(P=1.24 \times 10^{-6}\right)$; the latter locus was not identified in the single variant GWAS of severe COPD (Fig. 3).

\section{Emphysema}

In whole blood and lung tissue, we identified five genes significantly associated with \%LAA-950 and one gene with Perc15 (Table 3; Fig. 2). We found two significant associations of genes at loci previously associated with \%LAA-950, PSMA4 in 15q25 and ATF6B in 6p21, the latter which is located near $A G E R$. The top genomewide significant variant at this latter locus - which lies within the HLA (Human Leukocyte Antigen) region is a nonsynonymous variant in $A G E R$; however, $A G E R$ was not significant in either blood or lung $(P=0.81$ and 0.18 , respectively). LILRA3, DCBLD1, and ITGA1 are at loci not previously associated with COPD or emphysema.

\section{Validation in other reference transcriptome databases}

To provide further evidence for differentially expressed genes associated with severe COPD and emphysema, we repeated our analysis using additional reference transcriptome databases with the same GWAS data. In blood, we validated PSMA4, EGLN2, and RAB4B for severe COPD $\left(P=3.79 \times 10^{-14}, 1.34 \times 10^{-5}\right.$, and $1.33 \times 10^{-4}$, respectively), and PSMA4 and LILRA3 for \%LAA-950 ( $P=$ $3.37 \times 10^{-7}$ and $3.62 \times 10^{-5}$, respectively) by using GTExBlood as a validation for genes identified through whole blood transcriptome analysis (Table 3 ). We also validated WNT3 for severe COPD $\left(P=4.27 \times 10^{-6}\right)$ and DCBLD1 for \%LAA-950 $\left(P=1.41 \times 10^{-4}\right)$ for genes identified from 

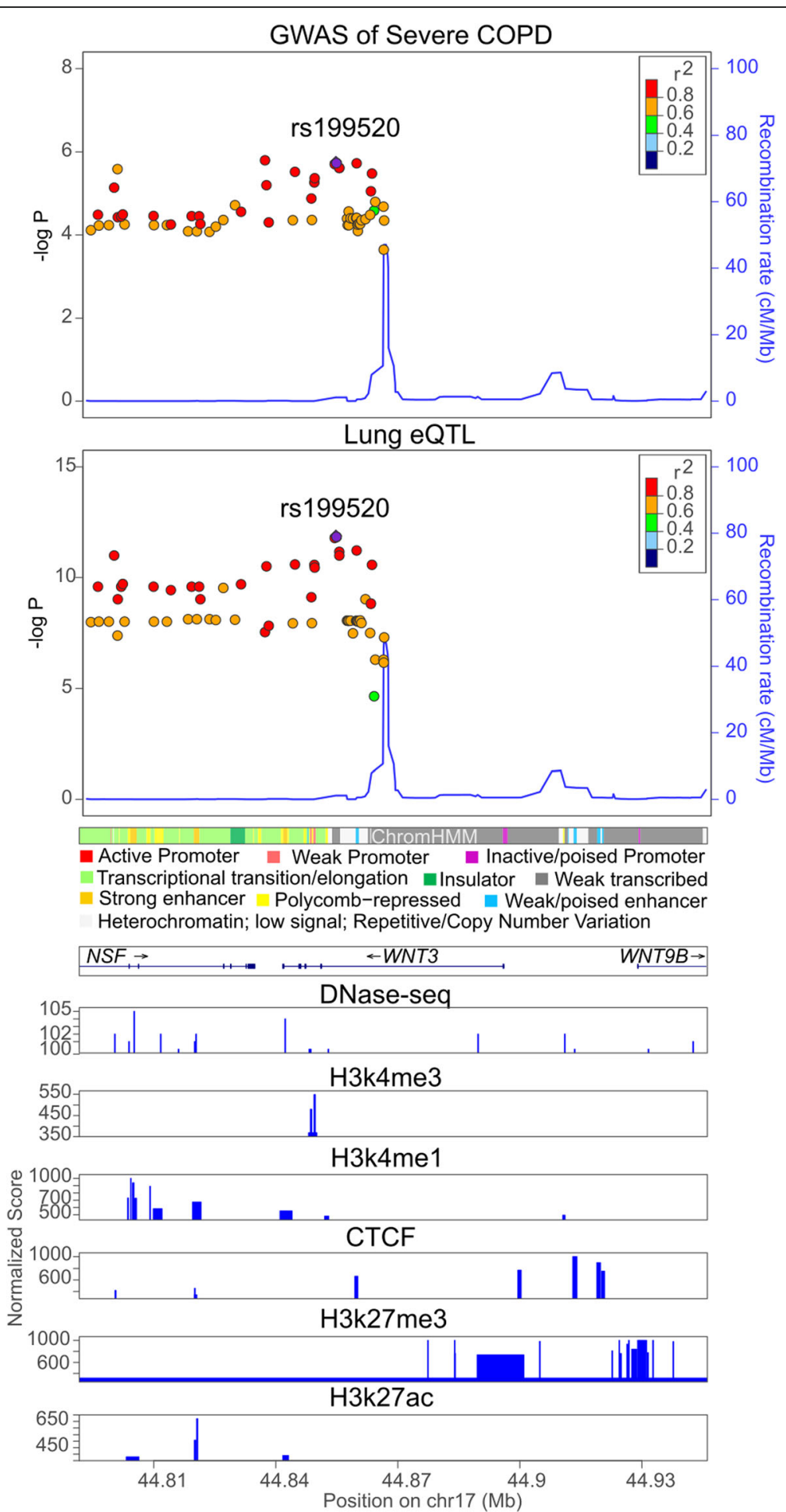

Fig. 3 Regional association plots within $50 \mathrm{~kb}$ of WNT3. GWAS of severe COPD and lung eQTL are shown in the upper panel. Chromatin states and epigenomic marks of normal human lung fibroblasts are shown in the lower panel (see Additional file 1: Supplementary Methods)

GTEx-Lung using a lung transcriptome database from Lung-eQTL Consortium (Table 3). We also noted that for several genes, a prediction model was not available, likely due to lower power and sample size in the validation dataset for whole blood [9]. Although the association of FAM13A was initially identified using blood dataset, its association was significant using Lung-eQTL Consoritium (Z score $\left.=4.52, P=6.3 \times 10^{-6}\right)$. 


\section{Colocalization analysis of validated genes}

Gene expression differences identified using SPrediXcan may be causally associated with the phenotype of interest, but also can be due to linkage disequilibrium (LD) [15]. To determine whether there was evidence of shared causality, we performed colocalization analysis, using a method that allows for multiple causal variants. Of the seven associations, six occupied at least one shared variant (Table 4): PSMA4, EGLN2, and WNT3 (Fig. 3) for severe COPD; PSMA4, LILRA3 (Additional file 1: Figure S1), and DCBLD1 (Additional file 1: Figure S2) for \%LAA-950. For associations identified in lung, we additionally confirmed the colocalization signals using the Lung-eQTL consortium dataset (Additional file 1: Table S1). We then sought to leverage functional annotation of shared variants especially for those with high colocalization probability. Some colocalized variants associated with PSMA4, LILRA3, DCBLD1, and WNT3 located in annotated regulatory regions (e.g., rs35061187 is in active transcription start site (TSS) in lung fibroblasts) or predicted to affect transcription factor binding (Additional file 1: Table S1 and S2).

\section{Genetically regulated differential expression of genes in known susceptibility loci}

Of the above significantly differentially regulated genes, four are in known susceptibility loci 4 q 22 and $15 \mathrm{q} 25$ with severe COPD, and 6p21 and 15q25 with \%LAA950). We also sought to investigate whether additional known susceptibility loci for severe COPD and quantitative emphysema affect the genetically regulated expression of nearby genes. We investigated nominal association results $(P<0.05)$ in other nine susceptibility loci in either discovery or validation datasets. Using this criterion, we found 5 additional suggestive associations, namely TGFB2 (1q41), HHIP (4q31), and RIN3 (14q32.12) with severe COPD, and HHIP (4q31) with \%LAA-950 and Perc15 (Additional file 1: Table S3). However, we did not find any suggestive signals in 11q22 (MMP12) with severe COPD, 14q32.13 (SERPINA10) with \%LAA-950, and 8p22 (DLC1) with \%LAA-950 and Perc15.

\section{Pathway enrichment analysis}

In contrast to genetic gene set enrichment methods that rely only on the location of the SNP to infer affected genes [17], we used the results of our predicted gene expression to identify pathways by using the top $1 \%$ of differentially expressed genes (Table 5, Additional file 1 : Supplementary Methods). We identified enrichment of the $\mathrm{T}$ cell receptor signaling pathway (corrected $P=$ $6.6 \times 10^{-3}$ ); this pathway included PSMA4 along with genes in the HLA complex. We also found significant enrichment for proteasome core complex genes (corrected $P=2.82 \times 10^{-2}$ ) which included PSMF1, PSMB4, and PSMB9. An additional pathway of interest was cellmatrix adhesion of collagen binding (corrected $P=$ $2.74 \times 10^{-3}$ ) (Table 5). We also found enrichment of the asthma pathway using the KEGG database (corrected $P=4.80 \times 10^{-3}$ ), containing MS4A2 and genes in HLA.

\section{Discussion}

Genome-wide association studies have arguably become the mainstay of identifying genetic risk factors for complex disease. However, these studies cannot identify which gene(s) in the region is responsible for the association, and testing all variants individually and independently is likely suboptimal. Here, we used an integrative method that combines the genetic component of gene expression with genetic association analysis in severe COPD and quantitative emphysema to predict differentially expressed genes. Importantly, this method focuses on the association of genetic component of gene expression, not gene expression as a whole, as is typical in most gene expression studies. We also provided additional support of our results by examining results in a second gene expression dataset, and performing colocalization analysis that attempts to identify whether association signals for gene expression and a phenotype of interest appear to be driven by the same causal variant(s). We implicated genes that are genetically

Table 4 Colocalized variants in validated genes and association statistics in corresponding GWAS and eQTL datasets

\begin{tabular}{|c|c|c|c|c|c|c|c|}
\hline Locus & Phenotype & Gene & Tissue & Top colocalized variant & Variant CLPP & $\begin{array}{l}\text { GWAS } \\
P \text { value }\end{array}$ & $\begin{array}{l}\text { eQTL } \\
P \text { value }\end{array}$ \\
\hline $6 q 22$ & \%LAA-950 & DCBLD1 & Lung & rs34882116 & 0.13 & 2.87E-05 & $1.28 \mathrm{E}-11$ \\
\hline $15 q 25$ & Severe COPD & PSMA4 & Blood & rs56077333 & 0.21 & 1.57E-18 & 4.16E-06 \\
\hline $15 q 25$ & $\%$ LAA-950 & PSMA4 & Blood & rs56077333 & 0.14 & 2.64E-09 & 4.16E-06 \\
\hline $17 q 21$ & Severe COPD & WNT3 & Lung & rs199520 & 0.21 & 1.75E-06 & 1.30E-12 \\
\hline $19 q 13$ & Severe COPD & EGLN2 & Blood & rs35755165 & 0.12 & 9.91E-09 & 1.87E-06 \\
\hline $19 q 13$ & $\%$ LAA-950 & LILRA3 & Blood & rs380267 & 0.28 & 3.32E-05 & 4.54E-47 \\
\hline
\end{tabular}

CLPP $=$ Colocalization posterior probability, the probability that a particular variant is causal in both GWAS and eQTL. A CLPP of 0.01 or greater previously demonstrated high accuracy and precision (see Additional file 1: Supplementary Methods), [15]. Only top colocalized variants, a variant with highest CLPP for each gene/tissue, are shown 
Table 5 Selected results of pathway enrichment analysis based on predicted differential gene expression

\begin{tabular}{|c|c|c|c|c|}
\hline Phenotype & Tissue & Functional category & $P$ value for enrichment* & Overlapping genes \\
\hline Severe COPD & Lung & $\begin{array}{l}\text { collagen binding involved in } \\
\text { cell-matrix adhesion }\end{array}$ & $2.70 \mathrm{E}-03$ & ITGA10, ITGA2, ITGA1 \\
\hline Severe COPD & Lung & proteasome core complex & $2.80 \mathrm{E}-02$ & PSMF1, PSMB4, PSMB9 \\
\hline Severe COPD & Lung & $\begin{array}{l}\text { translation factor activity, RNA } \\
\text { binding }\end{array}$ & 3.70E-02 & TCEB3, EEFSEC, TUFM, EIF3C, EIF3CL \\
\hline$\%$ LAA-950 & Whole Blood & MHC class II protein complex & $5.20 \mathrm{E}-05$ & $\begin{array}{l}\text { HLA-DQB1, HLA-DRB1, HLA-DQA1, HLA-DQB2, } \\
\text { HLA-DQA2 }\end{array}$ \\
\hline$\%$ LAA-950 & Whole Blood & PD-1 signaling & $6.30 \mathrm{E}-04$ & $\begin{array}{l}\text { HLA-DQB1, HLA-DRB1, HLA-DQA1, HLA-DQB2, } \\
\text { HLA-DQA2 }\end{array}$ \\
\hline \%LAA-950 & Whole Blood & Downstream TCR signaling & $8.00 \mathrm{E}-04$ & $\begin{array}{l}\text { PSMA4, NFKBIA, BCL10, HLA-DQB1, HLA-DRB1, } \\
\text { HLA-DQA1, HLA-DQB2, HLA-DQA2 }\end{array}$ \\
\hline$\%$ LAA-950 & Whole Blood & Asthma & $4.80 \mathrm{E}-03$ & $\begin{array}{l}\text { MS4A2, HLA-DQB1, HLA-DRB1, HLA-DQA1, } \\
\text { HLA-DQA2 }\end{array}$ \\
\hline$\%$ LAA-950 & Whole Blood & $\begin{array}{l}\text { T cell receptor signaling } \\
\text { pathway }\end{array}$ & $6.60 \mathrm{E}-03$ & $\begin{array}{l}\text { PSMA4, NFKBIA, BCL10, HLA-DRB1, HLA-DQA1, } \\
\text { PVRIG, HLA-DQB2, HLA-DQA2 }\end{array}$ \\
\hline Perc15 & Lung & $\begin{array}{l}\text { negative regulation of ERBB } \\
\text { signaling pathway }\end{array}$ & 4.50E-02 & NRG1, ITGA1, UBA52 \\
\hline
\end{tabular}

*P values were corrected for multiple testing using g:SCS method (see Additional file 1: Supplementary Methods)

regulated in known COPD-susceptibility loci, such as $F A M 13 A$, and also found genes in regions that were not previously reported: WNT3 for severe COPD, and $D C B L D 1$ and LILRA3 for quantitative emphysema.

We found a novel association of WNT3 in lung tissue with severe COPD in two gene expression datasets. Although variants surrounding this gene in the $17 \mathrm{q} 21$ locus were not genome-wide significant in our COPD analysis GWAS (Fig. 3), the top signal (rs9912530) is in strong LD with variants previously reported in GWAS of $\mathrm{FEV}_{1}[18,19]$, interstitial lung disease [20], and idiopathic pulmonary fibrosis [21] $\left(\mathrm{r}^{2}\right.$ with these previously described variants, 0.55-0.72). WNT3 (Wnt family member 3) encodes Wnt3, a critical component of the Wnt-beta-catenin-TCF signaling pathway [22] and a required signal for the apical ectodermal ridge in limb patterning [23]. Deficient WNT3 is associated with tetraamelia syndrome, a Mendelian disease characterized by an absence of all limbs. The top signal is also in strong LD with variants associated with various complex diseases such as Parkinson's disease and celiac disease $\left(\mathrm{r}^{2}\right.$ 0.72-0.79) [24, 25]. Previous expression studies of small airway epithelium found that this gene, along with its Wnt signaling companions, was down-regulated in smokers compared with nonsmokers [26]. Of interest, FAM13A, a well-supported COPD susceptibility gene, has been involved in the Beta-catenin/Wnt signaling pathway by protein degradation [27]. While there is substantial interest in Wnt signaling in lung disease [28], the contribution of WNT3 to the pathogenesis of COPD requires further investigation. To address whether these findings were specific for severe COPD, we repeated the analysis including moderate disease (GOLD 2). All of our genes were at least nominally significant, though overall the significance of our findings was attenuated (Additional file 1: Table S4).

For emphysema, we identified novel associations of LILRA3 and DCBLD1 using whole blood and lung tissue, respectively, and validated these findings in additional gene expression datasets. LILRA3 (leukocyte immunoglobulin like receptor A3) is a gene encoding a soluble receptor for class I major histocompatibility complex (MHC) antigens expressed in monocytes and B cells, which is located in the 19q13 locus. Our top hit from GWAS in this locus, was not genome-wide significant (rs384116 with $P=1.88 \times 10^{-5}$; Additional file 1: Figure S1), and 13-Mb away from the previously reported locus [16] that contains EGLN2 and RAB4B (rs7937; $\mathrm{r}^{2}$ 0.002). It is in modest LD with variants suggestively associated with $\mathrm{FEV}_{1} / \mathrm{FVC}$ [18] $\left(\mathrm{r}^{2}\right.$ 0.44), in strong LD with variants genome-wide significantly associated with HDL-C level [29] and prostate cancer [30] ( $r^{2}$ 0.92-0.99). Blood may be the most relevant tissue for this gene, as it is preferentially expressed [31] with a high estimate of heritability of gene expression in whole blood [32]. However, it may also have an effect in other tissues, given its broad eQTL effects identified by multi-tissue eQTL analysis [33]. This was supported by the suggestive signals of this gene using lung tissue in SPrediXcan analysis $\left(P=7.71 \times 10^{-5}\right.$ in GTEx-Lung and $1.38 \times 10^{-4}$ in the Lung-eQTL Consortium with the same direction of effect). Nonetheless, its functional role in COPD has not been described previously. Our other novel association identified in lung tissue, $D C B L D 1$ (discoidin, CUB and LCCL domain containing 1), located in the $6 q 22$ locus, is an integral component of cell membranes and binds to oligosaccharides [34]. GWAS signals in this locus 
are also sub-genome wide significant (Additional file 1: Figure S2). Our top GWAS variant at this locus was in LD with variants associated with lung cancer [35] $\left(\mathrm{r}^{2} 0.54\right)$.

In addition to novel associations, our study also provides insight into disease-associated genes in known COPD susceptibility loci. We identified six genes (FAM13A, GPRIN3, HYKK, PSMA4, EGLN2, and $R A B 4 B)$ in three known COPD-susceptibility loci for which their genetic component of gene expression in blood or in lung tissue is associated with severe COPD. Five of these six genes are not the most proximal to the top associated SNP, a phenomenon previously observed in other genetic association studies $[36,37]$. These findings underscore the complexity of genetic regulation in tissues and also identify multiple potential effector genes in the same locus. For example, in 15q25, PSMA4, and not CHRNA3 (the nearest gene to the top GWAS hit) was highlighted in S-PrediXcan and colocalization analysis. Although a role for IREB2 has been clearly demonstrated [38], our study suggested that other genes in the locus, particularly PSMA4 - a gene encoded for subunit of proteasome complex that acts in the proteolytic pathway [39], may also be of biologic importance.

At the 4q22 locus, an association for FAM13A identified using DGN-Blood was not validated in the GTEx-blood dataset. However, a significant but directionally opposite association was identified in the Lung-eQTL consortium dataset. To further explore this phenomenon, we examined individual SNP eQTL data from the Framingham Heart Study (FHS) blood, and the lung tissue from the Lung eQTL consortium (Additional file 1: Supplementary Methods). We confirmed that SNPs have opposite directions of effect in lung and blood (Additional file 1: Figure S3 and S4). This finding is consistent with prior reports describing significant and opposite tissue specific effects of eQTLs [33, 40, 41]. The interpretation of this phenomenon is not clear, but may be a result of pleiotropic effects of FAM13A [42, 43]. Of note, a recent analysis of emphysema-related gene expression in blood and lung tissue [44] found that the expression of genes in two tissues are often opposite; together, our findings highlight the tissue-specific genetic regulation of genes in COPD susceptibility loci. At the 19q13 locus, while both EGLN2 and $R A B 4 B$ were successfully validated, only GWAS and eQTL signals for EGLN2 colocalized. This genetic locus was associated with COPD [16] and smoking behavior [45]. Although the causal gene(s) in this region is unclear, methylation and expression studies support the role of EGLN2 in this region [46]. EGLN2 (egl-9 family hypoxia inducible factor 2) encodes an enzyme that regulate the degradation of alpha subunit of hypoxia inducible factor (HIF) [47]. Gene and protein expression of HIF-1 $\alpha$ is reduced in lung tissue samples from COPD patients [48].
Although $A T F 6 B$ (activating transcription factor 6 beta) and ITGA1 (integrin subunit alpha 1) were not successfully validated, we cannot rule out the possibility of false negatives due to differences between the transcriptome datasets used for validation, and they are potentially interesting candidates for COPD. ATF6B was implicated in the unfolded protein response (UPR) pathway during endoplasmic reticulum (ER) stress following cigarette smoke, and may contribute to lung inflammation in patients with COPD [49], while integrins were found to be involved in COPD through the mitogenactivated protein kinase (MAPK) pathway [50, 51]. This region also harbors variants associated with $\mathrm{FEV}_{1} / \mathrm{FVC}$ [52]. Decreased expression of ITGA1 was observed in the small airways of patients with low $\mathrm{FEV}_{1}$ [53].

Our analysis assesses only the genetic component of gene expression. We also investigated whether these genes were differentially expressed in COPD patients, in 464 blood samples from the COPDGene study [54], and 151 lung tissue samples [55] (Additional file 1: Supplementary Methods and Table S5-S8). These genes were not differentially expressed, with the exception of LILRA3, which was nominally significant with \%LAA-950 $(P=0.03)$. Given that the genetic component of gene expression was replicated, we believe that the genetic findings are robust, and speculate that these null findings could be due to nongenetic (i.e. environmental) perturbations that may occur downstream, or as a result of the genetic effects. In fact, in several cases measurements of mRNA or protein are actually opposite those predicted by genetic risk. For example, SERPINA1 risk alleles result in decreased levels and increased risk for COPD, yet average, alpha-1 levels in patients with COPD are actually elevated. Similarly, genetic variants in $A G E R$ and DSP affect transcript or protein levels opposite than what is measured in disease $[4,56,57]$. The mechanisms underlying our genetic findings, as well as $A G E R$ and $D S P$, that result in null or opposite direction effects requires further experimental investigation.

In addition to examination of individual loci, we applied pathway enrichment analysis to nominally significant differentially expressed genes in severe COPD and quantitative emphysema both in whole blood and lung tissue. This analysis identified enrichment of the $\mathrm{T}$ cell receptor signaling pathway in emphysema. This finding is consistent with reports that found antigen-specific $\mathrm{T}$ cell differentiation in lungs of patients with severe emphysema [58]. Our analysis using gProfileR does not assess of direction of effect, and the relative up- or down-regulation of specific genes in this pathway makes determination of direction difficult. To attempt to infer direction, we used Gene Set Enrichment Analysis (GSEA; [59]). In these results, the TCR signaling pathway and downstream TCR response were up-regulated, 
though these results were not statistically significant (Additional file 1: Table S9). Further study will be needed to determine the combined effects of COPD genetic susceptibility variants on $\mathrm{T}$ cell function and whether these explain some immune dysfunction seen in COPD [60, 61]. The finding of the enrichment of genes in the proteasome core complex further suggested a role of proteasome in COPD as described previously. Somewhat surprisingly, we observed enrichment of the asthma pathway in KEGG using genes identified in quantitative emphysema. This finding complements the description of substantial genetic correlation of COPD and asthma [4], and the presence of quantitative emphysema (or lung hyperinflation) in asthmatic patients [62].

Our study did not identify associations of genetically regulated differential expression of genes at some previously reported GWAS loci. Moreover, some of our identified associations in our discovery dataset were not successfully validated in a second transcriptome dataset. These findings indicate some of the limitations of our approach. First, as S-PrediXcan uses cis genetic variants as predictors for gene expression, variants that have lesser or no effect on transcript abundance or act in trans would not be detected by this approach [63]. Second, although most genetic variants implicated by GWAS are likely regulatory, only a minority of genetic loci are explained by existing eQTLs [64]. This may be due to lack of data in the appropriate tissue, cell type, or biologic conditions; or the heterogeneity of gene expression studies of bulk tissue. We may overcome these issues as more gene expression datasets and newer techniques such as single-cell gene expression profiling [65] become widely available. Moreover, issues such as cell type composition, sample collection methods, disease status, and differences in analytic methods also made the overlapping analysis challenging. Third, the number of genes available for an analysis depends on the power and sample size of the expression data used in constructing a gene expression prediction model $[8,9]$. Given the noisy and condition-specific nature of gene expression datasets, variants with small effects on gene expression may be undetectable at the sample sizes available. Additionally, the difference in sample size among transcriptome databases decreases our power to validate or discover more genes.

However, despite technical and population differences, most cis-eQTLs appear to be consistent between studies [66]. Therefore, despite in some cases a modest value of overall coefficient of correlation between predicted and measured gene expression, associations of the genetic component of gene expression as inferred by imputed gene expression have been successfully in identifying disease-associated genes that complement existing methods.

\section{Conclusions}

In conclusion, we found that genetic determinants of gene expression were associated with severe COPD and quantitative emphysema phenotypes, identifying genes at known loci, and identifying novel COPD-associated genes. These findings were obtained by integrating GWAS results with gene expression data, performing colocalization analysis, and validating key results in independent gene expression datasets. These findings may provide mechanistic insights into the genetics of COPD.

\section{Endnotes}

${ }^{1}$ A preliminary abstract of this study was previously published: Sakornsakolpat P, Morrow JD, Castaldi PJ, Hersh CP, Silverman EK, Manichaikul A, et al. Integrative Analysis of Genomics and Transcriptomics Identifies Association of PSMA4 with Emphysema. American Journal of Respiratory and Critical Care Medicine 2017;195:A7614. Available from: http://www.atsjournals.org.

\section{Additional file}

Additional file 1: Supplementary Methods. Table S1. Colocalization probability and regulatory annotations for colocalized variants using corresponding GWAS and Lung-eQTL consortium datasets (see Excel file). Table S2. Colocalization probability and regulatory annotations for colocalized variants using corresponding GWAS and GTEx eQTL (blood and lung) datasets (see Excel file). Table S3. Additional imputed gene expression association results at previously described GWAS significant loci. Table S4. Result of association analysis between imputed gene expression and moderate to severe COPD of reported genes from severe COPD. Table S5. Differential expression analysis between COPD and controls using blood RNA-seq in COPDGene. Table S6. Differential expression analysis of quantitative emphysema using blood RNA-seq in COPDGene. Table S7. Differential expression analysis between severe COPD and controls using lung tissues. Table S8. Differential expression analysis of \%LAA-950 and Perc15 using lung tissues. Table S9. T-cell-associated gene sets from Reactome using a ranked gene list from associations of \%LAA-950 (DGN-Blood). Table S10. Covariate adjustments for differential expression analysis. Figure S1. Regional association plots within 50kb of LILRA3. GWAS of \%LAA-950 and blood eQTL were shown in upper panel. Chromatin states and epigenomic marks of normal human lung fibroblast were shown in lower panel (see Supplementary Methods). Figure S2. Regional association plots within 50kb of DCBLD1. GWAS of \%LAA-950 and lung eQTL were shown in upper panel. Chromatin states and epigenomic marks of normal human lung fibroblast were shown in lower panel (see Supplementary Methods). Figure S3. Scatter plot of effect size of significant SNPs from eQTL studies of blood and lung tissue for FAM13A. Figure S4. Contribution of each FAM13A SNP in prediction models to overall association statistics. (ZIP $758 \mathrm{~kb}$ )

\section{Abbreviations}

\%LAA-950: The percentage low attenuation area at - $950 \mathrm{HU}$ threshold; CLPP: Colocalization posterior probability; COPD: Chronic obstructive pulmonary disease; COPDGene: Genetic Epidemiology of COPD; DGNBlood: Whole blood from Depression Genes and Networks; eCAVIAR: eQTL and GWAS Causal Variant Identification in Associated Regions; ECLIPSE: Evaluation of COPD Longitudinally to Identify Predictive Surrogate Endpoints; eQTL: Expression quantitative trait loci; ER: Endoplasmic reticulum; GenKOLS: Genetics of COPD, Norway; GTEx-Blood: Whole blood from Genotype-Tissue Expression consortium; GTEx-Lung: Lung tissue from Genotype-Tissue Expression consortium; GWAS: Genome-wide association studies; HLA: Human Leukocyte Antigen; HU: Hounsfield units; LD: Linkage 
disequilibrium; MAPK: Mitogen-activated protein kinase; MHC: Major histocompatibility complex; NAS: Normative Aging Study; NETT: National Emphysema Treatment Trial; Perc15: The HU at the 15th percentile of the density histogram; TSS: Transcription start site; UPR: Unfolded protein response

\section{Acknowledgements}

We would like to thank Drs. Per Bakke, Amund Gulsvik, David Sparrow, Augusto Litonjua, Ruth Tal-Singer, and Nick Locantore for use of the GWAS summary statistics. We thank Farhad Hormozdiari for his support on eCAVIAR and helpful discussions.

\section{Funding}

Supported by Prince Mahidol Award Youth Program Scholarship and Faculty of Medicine Siriraj Hospital Fellowship (P.S.); and NHLBI grants R01 HL113264, R01 HL086936, P01 HL114501 (M.H.C. and E.K.S.), R01 HL137927, R01 HL135142 (M.H.C.), and R01 HL131565 (A.M.). The COPDGene study (NCT00608764) is supported by NHLBI grants R01 HL089897 and R01 HL089856, and the COPD Foundation through contributions made to an Industry Advisory Board composed of AstraZeneca, Boehringer Ingelheim, Novartis, Pfizer, GlaxoSmithKline, Siemens, and Sunovion. The National Emphysema Treatment Trial was supported by NHLBI grants N01HR76101, N01HR76102, N01HR76103, N01HR76104, N01HR76105, N01HR76106, N01HR76107, N01HR76108, N01HR76109, N01HR76110, N01HR76111, N01HR76112, N01HR76113, N01HR76114, N01HR76115, N01HR76116, N01HR76118, and N01HR76119; the Centers for Medicare and Medicaid Services; and the Agency for Healthcare Research and Quality. The Norway GenKOLS study (Genetics of Chronic Obstructive Lung Disease, GSK code RES11080) and the ECLIPSE study (NCT00292552; GSK code SCO104960) were funded by GlaxoSmithKline. The funding body has no roles in the design of the study and collection, analysis, and interpretation of data and in writing the manuscript.

\section{Availability of data and materials}

The datasets used and/or analyzed during the current study are available from the corresponding author on reasonable request.

\section{Authors' contributions}

Study design: PS, EKS and MHC. Data collection: YB, EKS, MHC. Data analysis: PS, YB, MHC. Critical revision of the manuscript: all authors. All authors read and approved the final manuscript.

\section{Ethics approval and consent to participate}

Local institutional review boards provided ethical approval for the clinical centers. Written informed consent was obtained in all studies.

\section{Consent for publication}

Not applicable

\section{Competing interests}

The authors declare that they have no competing interests.

\section{Publisher's Note}

Springer Nature remains neutral with regard to jurisdictional claims in published maps and institutional affiliations.

\footnotetext{
Author details

${ }^{1}$ Channing Division of Network Medicine, Department of Medicine, Brigham and Women's Hospital, 181 Longwood Avenue, Room 451, Boston, MA 02115, USA. ${ }^{2}$ Department of Medicine, Faculty of Medicine Siriraj Hospital, Mahidol University, Bangkok, Thailand. Division of General Medicine, Department of Medicine, Brigham and Women's Hospital, 181 Longwood Avenue, Room 451, Boston, MA 02115, USA. ${ }^{4}$ Division of Pulmonary and Critical Care Medicine, Department of Medicine, Brigham and Women's Hospital, 181 Longwood Avenue, Room 451, Boston, MA 02115, USA. ${ }^{5}$ Department of Molecular Medicine, Institut universitaire de cardiologie et de pneumologie de Québec, Laval University, Quebec, Canada. 'Department of Public Health Sciences, Center for Public Health Genomics and Biostatistics Section, University of Virginia, Charlottesville, VA, USA.
}

Received: 9 January 2018 Accepted: 6 March 2018

Published online: 22 March 2018

\section{References}

1. Zhou JJ, Cho MH, Castaldi PJ, Hersh CP, Silverman EK, Laird NM. Heritability of chronic obstructive pulmonary disease and related phenotypes in smokers. Am J Respir Crit Care Med. 2013;188:941-7.

2. Vogelmeier CF, Criner GJ, Martinez FJ, Anzueto A, Barnes PJ, Bourbeau J, et al. Global strategy for the diagnosis, management, and prevention of chronic obstructive lung disease 2017 report. GOLD executive summary. Am J Respir Crit Care Med. 2017;195:557-82.

3. Cho MH, McDonald M-LLN, Zhou X, Mattheisen M, Castaldi PJ, Hersh CP, et al. Risk loci for chronic obstructive pulmonary disease: a genome-wide association study and meta-analysis. Lancet Respir Med. 2014;2:214-25.

4. Hobbs BD, de Jong K, Lamontagne M, Bosse Y, Shrine N, Artigas MS, et al. Genetic loci associated with chronic obstructive pulmonary disease overlap with loci for lung function and pulmonary fibrosis. Nat Genet. 2017;49:426-32.

5. Cho MH, Castaldi PJ, Hersh CP, Hobbs BD, Barr RG, Tal-Singer R, et al. A genome-wide association study of emphysema and airway quantitative imaging phenotypes. Am J Respir Crit Care Med. 2015;192:559-69.

6. Hindorff LA, Sethupathy P, Junkins HA, Ramos EM, Mehta JP, Collins FS, et al. Potential etiologic and functional implications of genome-wide association loci for human diseases and traits. Proc Natl Acad Sci U S A. 2009;106:9362-7.

7. Nicolae DL, Gamazon E, Zhang W, Duan S, Dolan ME, Cox NJ. Traitassociated SNPs are more likely to be eQTLs: annotation to enhance discovery from GWAS. PLoS Genet. 2010;6:e1000888.

8. Gamazon ER, Wheeler HE, Shah KP, Mozaffari SV, Aquino-Michaels K, Carroll $\mathrm{RJ}$, et al. A gene-based association method for mapping traits using reference transcriptome data. Nat Genet. 2015;47:1091-8.

9. Gusev A, Ko A, Shi H, Bhatia G, Chung W, Penninx BWJH, et al. Integrative approaches for large-scale transcriptome-wide association studies. Nat Genet. 2016;48:245-52.

10. Barbeira A, Dickinson SP, Torres JM, Torstenson ES, Zheng J, Wheeler HE, et al. Integrating tissue specific mechanisms into GWAS summary results. bioRxiv. 2017.

11. Visscher PM, Wray NR, Zhang Q, Sklar P, McCarthy MI, Brown MA, et al. 10 years of GWAS discovery: biology, function, and translation. Am J Hum Genet. 2017;101:5-22.

12. Zhou X, Baron RM, Hardin M, Cho MH, Zielinski J, Hawrylkiewicz I, et al. Identification of a chronic obstructive pulmonary disease genetic determinant that regulates HHIP. Hum Mol Genet. 2012;21:1325-35.

13. Flister MJ, Tsaih S-W, O'Meara CC, Endres B, Hoffman MJ, Geurts AM, et al. Identifying multiple causative genes at a single GWAS locus. Genome Res. 2013:23:1996-2002.

14. Claussnitzer M, Hui C-C, Kellis M. FTO obesity variant and adipocyte Browning in humans. N Engl J Med. 2016:374:192-3.

15. Hormozdiari F, van de Bunt M, Segrè AV, Li X, Joo JWJ, Bilow M, et al. Colocalization of GWAS and eQTL signals detects target genes. Am J Hum Genet. 2016;99:1245-60.

16. Cho MH, Castaldi PJ, Wan ES, Siedlinski M, Hersh CP, Demeo DL, et al. A genome-wide association study of COPD identifies a susceptibility locus on chromosome 19q13. Hum Mol Genet. 2012;21:947-57.

17. Liu JZ, McRae AF, Nyholt DR, Medland SE, Wray NR, Brown KM, et al. A versatile gene-based test for genome-wide association studies. Am J Hum Genet 2010:87:139-45.

18. Lutz SM, Cho MH, Young K, Hersh CP, Castaldi PJ, McDonald M-LL, et al. A genome-wide association study identifies risk loci for spirometric measures among smokers of European and African ancestry. BMC Genet. 2015:16:138.

19. Wain LV, Shrine N, Miller S, Jackson VE, Ntalla I, Soler Artigas M, et al. Novel insights into the genetics of smoking behaviour, lung function, and chronic obstructive pulmonary disease (UK BiLEVE): a genetic association study in UK biobank. Lancet Respir Med. 2015;3:769-81.

20. Fingerlin TE, Murphy E, Zhang W, Peljto AL, Brown KK, Steele MP, et al. Genome-wide association study identifies multiple susceptibility loci for pulmonary fibrosis. Nat Genet. 2013:45:613-20.

21. Noth I, Zhang Y, Ma S-F, Flores C, Barber M, Huang Y, et al. Genetic variants associated with idiopathic pulmonary fibrosis susceptibility and mortality: a genome-wide association study. Lancet Respir Med. 2013;1:309-17.

22. Clevers H, Nusse R. Wnt/beta-catenin signaling and disease. Cell. 2012;149: 1192-205. 
23. Barrow JR, Thomas KR, Boussadia-Zahui O, Moore R, Kemler R, Capecchi MR, et al. Ectodermal Wnt3/beta-catenin signaling is required for the establishment and maintenance of the apical ectodermal ridge. Genes Dev. 2003;17:394-409.

24. Hill-Burns EM, Wissemann WT, Hamza TH, Factor SA, Zabetian CP, Payami H. Identification of a novel Parkinson's disease locus via stratified genomewide association study. BMC Genomics. 2014;15:118.

25. Dubois PCA, Trynka G, Franke L, Hunt KA, Romanos J, Curtotti A, et al. Multiple common variants for celiac disease influencing immune gene expression. Nat Genet. 2010;42:295-302.

26. Wang R, Ahmed J, Wang G, Hassan I, Strulovici-Barel Y, Hackett NR, et al. Down-regulation of the canonical Wnt beta-catenin pathway in the airway epithelium of healthy smokers and smokers with COPD. PLoS One. 2011;6: e14793.

27. Jiang Z, Lao T, Qiu W, Polverino F, Gupta K, Guo F, et al. A chronic obstructive pulmonary disease susceptibility gene, FAM13A, regulates protein stability of beta-catenin. Am J Respir Crit Care Med. 2016;194: 185-97.

28. Baarsma HA, Königshoff M. "WNT-er is coming": WNT signalling in chronic lung diseases. Thorax. 2017;72:746-59.

29. Spracklen CN, Chen P, Kim YJ, Wang X, Cai H, Li S, et al. Association analyses of east Asian individuals and trans-ancestry analyses with European individuals reveal new loci associated with cholesterol and triglyceride levels. Hum Mol Genet. 2017;26:1770-84.

30. Xu J, Mo Z, Ye D, Wang M, Liu F, Jin G, et al. Genome-wide association study in Chinese men identifies two new prostate cancer risk loci at 9q31.2 and 19q13.4. Nat Genet. 2012:44:1231-5.

31. Melé M, Ferreira PG, Reverter F, DeLuca DS, Monlong J, Sammeth M, et al. Human genomics. The human transcriptome across tissues and individuals. Science. 2015;348:660-5.

32. Wright FA, Sullivan PF, Brooks Al, Zou F, Sun W, Xia K, et al. Heritability and genomics of gene expression in peripheral blood. Nat Genet. 2014;46:430-7.

33. Consortium GTe. Human genomics. The genotype-tissue expression (GTEx) pilot analysis: multitissue gene regulation in humans. Science. 2015;348:648-60.

34. Reference Genome Group of the Gene Ontology Consortium. The Gene Ontology's Reference Genome Project: a unified framework for functional annotation across species. PLoS Comput Biol. 2009;5:e1000431.

35. Lan Q, Hsiung CA, Matsuo K, Hong Y-CC, Seow A, Wang Z, et al. Genomewide association analysis identifies new lung cancer susceptibility loci in never-smoking women in Asia. Nat Genet. 2012;44:1330-5.

36. Maurano MT, Humbert R, Rynes E, Thurman RE, Haugen E, Wang $H$, et al. Systematic localization of common disease-associated variation in regulatory DNA. Science. 2012;337:1190-5.

37. Shooshtari $P$, Huang $H$, Cotsapas $C$. Integrative genetic and epigenetic analysis uncovers regulatory mechanisms of autoimmune disease. Am J Hum Genet. 2017;101:75-86.

38. Cloonan SM, Glass K, Laucho-Contreras ME, Bhashyam AR, Cervo M, Pabón MA, et al. Mitochondrial iron chelation ameliorates cigarette smoke-induced bronchitis and emphysema in mice. Nat Med. 2016;22:163-74.

39. Weathington NM, Sznajder Jl, Mallampalli RK. The emerging role of the ubiquitin proteasome in pulmonary biology and disease. Am J Respir Crit Care Med. 2013;188:530-7.

40. Fu J, Wolfs MGM, Deelen P, Westra H-J, Fehrmann RSN, Te Meerman GJ, et al. Unraveling the regulatory mechanisms underlying tissue-dependent genetic variation of gene expression. PLoS Genet. 2012;8:e1002431.

41. Hauberg ME, Zhang W, Giambartolomei C, Franzén O, Morris DL, Vyse TJ, et al. Large-scale identification of common trait and disease variants affecting gene expression. Am J Hum Genet. 2017;100:885-94.

42. Jiang Z, Knudsen NH, Wang G, Qiu W, Naing ZZC, Bai Y, et al. Genetic control of fatty acid $\beta$-oxidation in chronic obstructive pulmonary disease. Am J Respir Cell Mol Biol. 2017;56:738-48.

43. Ligthart S, Vaez A, Hsu Y-H. Inflammation working group of the CHARGE consortium, PMI-WG-XCP, LifeLines cohort study, et al. bivariate genomewide association study identifies novel pleiotropic loci for lipids and inflammation. BMC Genomics. 2016;17:443.

44. Obeidat M, Nie Y, Fishbane N, Li X, Bossé Y, Joubert P, et al. Integrative Genomics of Emphysema Associated Genes Reveals Potential Disease Biomarkers. Am J Respir Cell Mol Biol. 2017.

45. Tobacco and Genetics Consortium. Genome-wide meta-analyses identify multiple loci associated with smoking behavior. Nat Genet. 2010;42:441-7.
46. Nedeljkovic I, Lahousse L, Carnero-Montoro E, Faiz A, Vonk JM, de Jong K, et al. COPD GWAS variant at 19q13.2 in relation with DNA methylation and gene expression. Hum Mol Genet. 2018;27:396-405.

47. Epstein AC, Gleadle JM, McNeill LA, Hewitson KS, O'Rourke J, Mole DR, et al. C. Elegans EGL-9 and mammalian homologs define a family of dioxygenases that regulate HIF by prolyl hydroxylation. Cell. 2001;107:43-54.

48. Yasuo M, Mizuno S, Kraskauskas D, Bogaard HJ, Natarajan R, Cool CD, et al. Hypoxia inducible factor-1a in human emphysema lung tissue. Eur Respir J. 2011:37:775-83.

49. Kelsen SG. The unfolded protein response in chronic obstructive pulmonary disease. Ann am Thorac Soc. 2016;13(Suppl 2):S138-45.

50. Renda T, Baraldo S, Pelaia G, Bazzan E, Turato G, Papi A, et al. Increased activation of p38 MAPK in COPD. Eur Respir J. 2008:31:62-9.

51. Ravanti L, Heino J, López-Otín C, Kähäri VM. Induction of collagenase-3 (MMP-13) expression in human skin fibroblasts by three-dimensional collagen is mediated by p38 mitogen-activated protein kinase. J Biol Chem. 1999;274:2446-55.

52. Wain LV, Shrine N, Artigas MS, Erzurumluoglu AM, Noyvert B, Bossini-Castillo $L$, et al. Genome-wide association analyses for lung function and chronic obstructive pulmonary disease identify new loci and potential druggable targets. Nat Genet. 2017:49:416-25.

53. Gosselink JV, Hayashi S, Elliott WM, Xing L, Chan B, Yang L, et al. Differential expression of tissue repair genes in the pathogenesis of chronic obstructive pulmonary disease. Am J Respir Crit Care Med. 2010;181:1329-35.

54. Parker MM, Chase RP, Lamb A, Reyes A, Saferali A, Yun JH, et al. RNA sequencing identifies novel non-coding RNA and exon-specific effects associated with cigarette smoking. BMC Med Genet. 2017;10:58.

55. Morrow JD, Zhou X, Lao T, Jiang Z, DeMeo DL, Cho MH, et al. Functional interactors of three genome-wide association study genes are differentially expressed in severe chronic obstructive pulmonary disease lung tissue. Sci Rep. 2017:7:44232.

56. Mathai SK, Pedersen BS, Smith K, Russell P, Schwarz MI, Brown KK, et al. Desmoplakin variants are associated with idiopathic pulmonary fibrosis. Am J Respir Crit Care Med. 2016;193:1151-60.

57. Cheng DT, Kim DK, Cockayne DA, Belousov A, Bitter H, Cho MH, et al. Systemic soluble receptor for advanced glycation endproducts is a biomarker of emphysema and associated with AGER genetic variants in patients with chronic obstructive pulmonary disease. Am J Respir Crit Care Med. 2013;188:948-57

58. Sullivan AK, Simonian PL, Falta MT, Mitchell JD, Cosgrove GP, Brown KK, et al. Oligoclonal CD4+ T cells in the lungs of patients with severe emphysema. Am J Respir Crit Care Med. 2005;172:590-6.

59. Subramanian A, Tamayo P, Mootha VK, Mukherjee S, Ebert BL, Gillette MA et al. Gene set enrichment analysis: a knowledge-based approach for interpreting genome-wide expression profiles. Proc Natl Acad Sci U S A. 2005;102:15545-50.

60. Bhat TA, Panzica L, Kalathil SG, Thanavala Y. Immune dysfunction in patients with chronic obstructive pulmonary disease. Ann Am Thorac Soc. 2015; 12(Suppl 2):S169-75.

61. Grundy S, Plumb J, Lea S, Kaur M, Ray D, Singh D. Down regulation of T cell receptor expression in COPD pulmonary CD8 cells. PLoS One. 2013;8: e71629.

62. Biernacki W, Redpath AT, Best JJ, MacNee W. Measurement of CT lung density in patients with chronic asthma. Eur Respir J. 1997;10:2455-9.

63. Sun W, Kechris K, Jacobson S, Drummond MB, Hawkins GA, Yang J, et al. Common genetic polymorphisms influence blood biomarker measurements in COPD. PLoS Genet. 2016;12:-e1006011.

64. Chun S, Casparino A, Patsopoulos NA, Croteau-Chonka DC, Raby BA, De Jager $\mathrm{PL}$, et al. Limited statistical evidence for shared genetic effects of eQTLs and autoimmune-disease-associated loci in three major immune-cell types. Nat Genet. 2017;49:600-5.

65. Petretto E. Single cell expression quantitative trait loci and complex traits. Genome med. 2013;5:72. Available from: https://doi.org/10.1186/gm476

66. Joehanes R, Zhang X, Huan T, Yao C, Ying S-X, Nguyen QT, et al. Integrated genome-wide analysis of expression quantitative trait loci aids interpretation of genomic association studies. Genome Biol. 2017;18:16. 